\title{
DETERMINAÇÃO DA CONCENTRAÇÃO LETAL MÉDIA (CL 50) DE BEAUVERIA BASSIANA PARA O CONTROLE DE BREVICORYNE BRASSICAE
}

\author{
DETERMINATION OF THE MEDIUM LETHAL CONCENTRATION (CL 50) OF \\ BEAUVERIA BASSIANA FOR THE CONTROL OF BREVICORYNE BRASSICAE
}

\author{
Gustavo Dias de Almeida ${ }^{1}$; Dirceu Pratissoli ${ }^{1}$; Ricardo Antônio Polanczyk ${ }^{1}$; \\ Anderson Mathias Holtz ${ }^{1}$; Victor Bernardo Vicentini ${ }^{1}$
}

\begin{abstract}
RESUMO
O pulgão da couve Brevicoryne brassicae (L.), é uma das principais pragas das Brássicas em geral, e a procura por alimentos mais saudáveis tem estimulado o desenvolvimento de técnicas dentro do manejo sustentável, como o controle microbiano. Este trabalho teve por objetivo determinar concentrações do fungo B. bassiana para o manejo alternativo do pulgão da couve. Ninfas de $B$. brassicae foram inoculadas em folhas de repolho imersas em suspensões fúngicas, do produto comercial Boveril ${ }^{\circledR}$, nas concentrações de $0,05,0,08,0,12,0,31,0,50,1,0,1,5,2,0$ gramas por litro de água destilada mais espalhante adesivo Hai-Teem ${ }^{\circledR}, \mathrm{e}$ mantidos a $26,0 \pm 1,0^{\circ} \mathrm{C}$ e UR de $75,0 \pm 5 \%$. Houve um acréscimo da mortalidade em função do aumento da concentração, pois, a menor concentração $(0,05 \mathrm{~g} / \mathrm{L})$ apresentou reduzido efeito de controle $14 \%$ de mortalidade confirmada. No entanto, as concentrações de 1,0 a 2,0g/L de água proporcionou uma mortalidade superior a 85\%. O valor encontrado para a CL50 foi de $0,233 \mathrm{~g} / \mathrm{L}$. Dessa forma o controle microbiano do pulgão da couve pode ser econômica e biologicamente viável para o manejo dessa praga em cultivos de brássicas.
\end{abstract}

Palavras chave: Controle microbiano, entomopatógeno, manejo integrado, CL 50.

\begin{abstract}
The cabbage aphid is one of the main curses of Brássicas in general, and the search for healthier foods has been stimulating the development of techniques inside of the maintainable handling, as the microbial control. The objective of this work was determine concentrations of the microbial B. bassiana for the handling of the cabbage aphid. Nymphs of B. brassicae were inoculated in submerged cabbage leaves in fungic suspensions, of the commercial product Boveril ${ }^{\circledR}$, in the concentrations of $0.05,0.08,0.12,0.31,0.50,1.0,1.5,2.0$ grams per liter of distilled water plus adhesive espalhante Hai-Teem $\circledast$, and maintained $26.0 \pm 1.0^{\circ} \mathrm{C}$ and $U R$ of $75.0 \pm 5 \%$. There was an increment of the mortality in function of the increase of the concentration, because, to smallest concentration $(0.05 \mathrm{~g} / \mathrm{L})$ it presented reduced effect of control $14 \%$ of confirmed mortality. However, the concentrations of $1.0-2.0 \mathrm{~g} / \mathrm{L}$ of water provided a superior mortality of $85 \%$. The value found for CL50 was of $0.233 \mathrm{~g} / \mathrm{L}$. In that way the microbial control of the cabbage aphid can be economical and biologically viable for the handling of this pest in brássicas cultivations.
\end{abstract}

Key words: Control microbial, entomopathogenic fungus, integrated handling, CL 50.

\section{INTRODUÇÃO}

O pulgão-da-couve, Brevicoryne brassicae (L.), encontra-se amplamente distribuído nas regiões temperadas e sub-tropicais do mundo, sendo que a quase totalidade de suas plantas hospedeiras pertence à família Brassicaceae (Ellis \& Singh, 1993). No Brasil, é considerado como uma das pragas mais prejudiciais a essas hortaliças (Longhini \& Busoli, 1993), causando grandes prejuízos devido à sucção

1 UFES/CCA, Laboratório de Entomologia, Dep ${ }^{\text {to }}$ Fitotecnia, Alto Universitário, C. postal 16. Alegre-ES; E-mail: gustavo. ccaufes@hotmail.com.

Fecha de Recepción: 08 Febrero 2007

Fecha de Aceptación: 05 Abril 2007 
contínua de seiva e introdução de toxinas no sistema vascular das plantas, além da transmissão de viroses (Catie, 1990) e o engruvinhamento das folhas em que se alojam, prejudicando o desenvolvimento da planta (Gallo et al., 2002).

Geralmente o controle dessa praga é realizado principalmente por aplicações de produtos químicos (Andrei, 1996). No entanto, o uso intensivo desses produtos tem causado diversos problemas, entre eles, a resistência dessas e outras pragas a inseticidas, exigindo um aumento da concentração e número de aplicações. Esse fato contribui para contaminação do solo, das plantas, da água, do homem e todos os microrganismos vivos e inimigos naturais que fazem parte do agrossistema. Esses problemas têm reforçado a necessidade de adoção de um manejo mais racional, como o uso do controle biológico com fungos entomopatogênicos (Robb \& Bittencourt, 1998).

Alves \& Almeida (1997) citam que este tipo de controle não é poluente, não provoca desequilíbrios biológicos, é duradouro e aproveita o potencial biótico do agrossistema. Além disso, não é tóxico para o homem e animais e pode ser aplicado com as máquinas convencionais, com pequenas adaptações. Alguns fungos entomopatogênicos, tais como Beauveria bassiana e Metarhizium anisopliae, têm demonstrado eficiência e outras vantagens sobre os produtos químicos no controle de pragas.

Nos EUA vem sendo comercializado o produto Naturalis-L ${ }^{\circledR}$, de B. bassiana, para o controle de Tetranychus urticae Koch (Acari: Tetranychidae). No Brasil, o produto Boveril ${ }^{\circledR}$, também formulado com este fungo, tem se mostrado efetivo no manejo de pragas agrícolas. Essa formulação foi desenvolvida após criteriosos estudos envolvendo a seleção de isolados, avaliação da virulência das estruturas infectivas de seu ciclo biológico (conídio aéreo, blastósporo e célula de levedura), toxicidade de produtos fitossanitários e de estratégias de uso (Alves et al., 2006).

O uso de entomopatógenos para o controle de pragas pode-se constituir em uma alternativa futura para solucionar os problemas de resistência (Omoto et al., 1994) e de contaminação ambiental tornando o agroecossistema hortícola, sustentável econômica e biologicamente (Alves et al., 2004). Com base no exposto, o presente trabalho teve por objetivo determinar concentrações do fungo $B$. bassiana para o manejo do B. brassicae, em condições de laboratório.

\section{MATERIAIS E MÉTODOS}

O experimento foi realizado no Laboratório de Entomologia do Núcleo de Desenvolvimento Científico e Tecnológico em Manejo Fitossanitário de Pragas e Doenças (NUDEMAFI) do Centro de Ciências Agrárias da Universidade Federal do Espírito Santo (CCA-UFES), em Alegre - ES.

Para realização dos experimentos, foram coletadas ninfas de B. brassicae em plantios de Brássicas na área experimental do (CCA-UFES) e posteriormente transferidos para plantas de repolho (Brassica oleracea L. var. capitata L.) cultivadas em sacolas plásticas $(16 \times 34 \mathrm{~cm})$ e mantidas em casa de vegetação.

O isolado de $B$. bassiana foi obtido através do produto comercial Boveril ${ }^{\circledR}$, cuja recomendação é de 500 milhões de conídios viáveis por grama do produto.

Para determinação da concentração letal, prepararam-se suspensões fúngicas nas concentrações de $0,05,0,08,0,12,0,31,0,50,1,0,1,5,2,0$ gramas por litro de água destilada mais espalhante adesivo Hai-Teem ${ }^{\circledR}$. Como testemunha utilizou-se apenas água destilada mais espalhante adesivo.

Folhas de repolho foram lavadas com água destilada, imersas em álcool 100\% por 1 minutos e novamente lavadas em água destilada, para a eliminação de agentes patogênicos externos. Após esses procedimentos, as folhas foram imersas nas suspensões fúngicas por 1 minuto e em seguida expostas ao ambiente por 30 minutos para secar o excesso de solução. Após a secagem, foram acondicionadas em placas de Petri $(15 \mathrm{~cm}$ de diâmetro e $5 \mathrm{~cm}$ de altura), forradas com papel absorvente e seus pecíolos envoltos com algodão úmido. Em cada placa de Petri foram liberadas 20 ninfas do B. brassicae. As placas de Petri foram levadas para estufa incubadora B.O.D à temperatura de $26,0 \pm 1,0^{\circ} \mathrm{C}$ e umidade relativa de $75,0 \pm 5 \%$.

As avaliações foram realizadas cinco dias depois, sendo considerados como mortos os insetos que não foram capazes de caminhar sobre a folha. Os insetos mortos de cada repetição foram acondicionados em gearbox ( $5 \mathrm{~cm}$ de diâmetro) forradas com algodão úmido e papel filtro. Após cinco dias em câmara úmida avaliou-se a mortalidade confirmada confirmada, pela esporulação do fungo.

O delineamento experimental foi inteiramente casualizado com 9 tratamentos e 5 repetições, sendo cada placa de Petri uma repetição. 
A eficiência do isolado foi avaliada através da percentagem de mortalidade de ninfas e CL50. A determinação da CL50 foi efetuada mediante a utilização do programa computacional Micro Probit (versão 3.0).

\section{RESULTADOS E DISCUSSÃO}

A menor concentração de B. bassiana $(0,05 \mathrm{~g} /$ L) apresentou $14 \%$ de mortalidade confirmada do pulgão até o quinto dia após a inoculação. As concentrações intermediárias $(0,08$ a $0,5 \mathrm{~g} / \mathrm{L})$, nesses mesmo período, causaram mortalidades confirmadas em torno de $30 \%$ a $60 \%$, e as maiores concentrações $(1,0$ a $2,0 \mathrm{~g} / \mathrm{L})$ entre 80 a $90 \%$. O valor da mortalidade aumentou aproximadamente 2,21 vezes quando a concentração passou de 0,05 para $0,08 \mathrm{~g} / \mathrm{L}$ (14 e $31 \%$, respectivamente). A partir de $0,12 \mathrm{~g} / \mathrm{L}$ o incremento na mortalidade pelo aumento da concentração do fungo foi menor, atingindo até $85 \%$ de mortalidade com 1,0 g/L. No entanto, com o dobro dessa concentração $(2,0 \mathrm{~g} / \mathrm{L}) \mathrm{o}$ incremento na mortalidade não foi expressivo, aumentando apenas 7,05 \% em relação à concentração de 1,0g/L (Gráfico 1).

A equação que melhor explica os resultados é Y= 5,894 + 1,415Log X, apresentando um $\mathrm{X}^{2}$ calculado de 5,7495, obtendo-se uma CL 50 de $0,233 \mathrm{~g} / \mathrm{L}$ de água. Apenas as concentrações de 0,05 , 0,08 e $0,12 \mathrm{~g} / \mathrm{L}$ resultaram em uma mortalidade confirmada inferior à CL 50.
Resultados semelhantes foram observados por Loureiro \& Moino Jr.(2006), que constataram a eficiência de $B$. bassiana na infecção do pulgão Aphis gossypii Glover e Myzus persicae (Sulzer) (Hemiptera: Aphididae), atingindo até $100 \%$ de mortalidade confirmada aos 7 dias.

Considerando a possibilidade de controle desta praga com pulverizações de B. bassiana, de forma semelhante ao uso de inseticidas químicos, as concentrações menos eficientes $(0,31$ e $0,50 \mathrm{~g} / \mathrm{L})$ apresentam-se como as mais viáveis economicamente. Pois, as menores concentrações possibilitam que um número maior de pulverizações seja realizado para o controle do pulgão ao mesmo custo econômico de uma aplicação com concentrações elevadas $(1,0$ a 2,0 g/L). Isso torna-se muito importante porque os fungos entomopatogenicos são, em sua maioria, extremamente suscetíveis a ação dos fatores ambientais adversos (alta temperatura, baixa umidade relativa e alta incidência de UV) que podem ocorrer após cada pulverização, comprometendo desta maneira sua sobrevivência no ambiente, e como consequiência, a eficiência da pulverização. Neste caso, o investimento de controle da praga com o fungo não é concentrado em uma única pulverização (com concentrações elevadas), mas diluído em diversas aplicações em baixas concentrações, aumentando as chances de sucesso, de forma a atender a demanda crescente por medidas não químicas de controle, de maneira semelhante ao que vem ocorrendo em outros cultivos agrícolas no país (Alves et al., 2001).

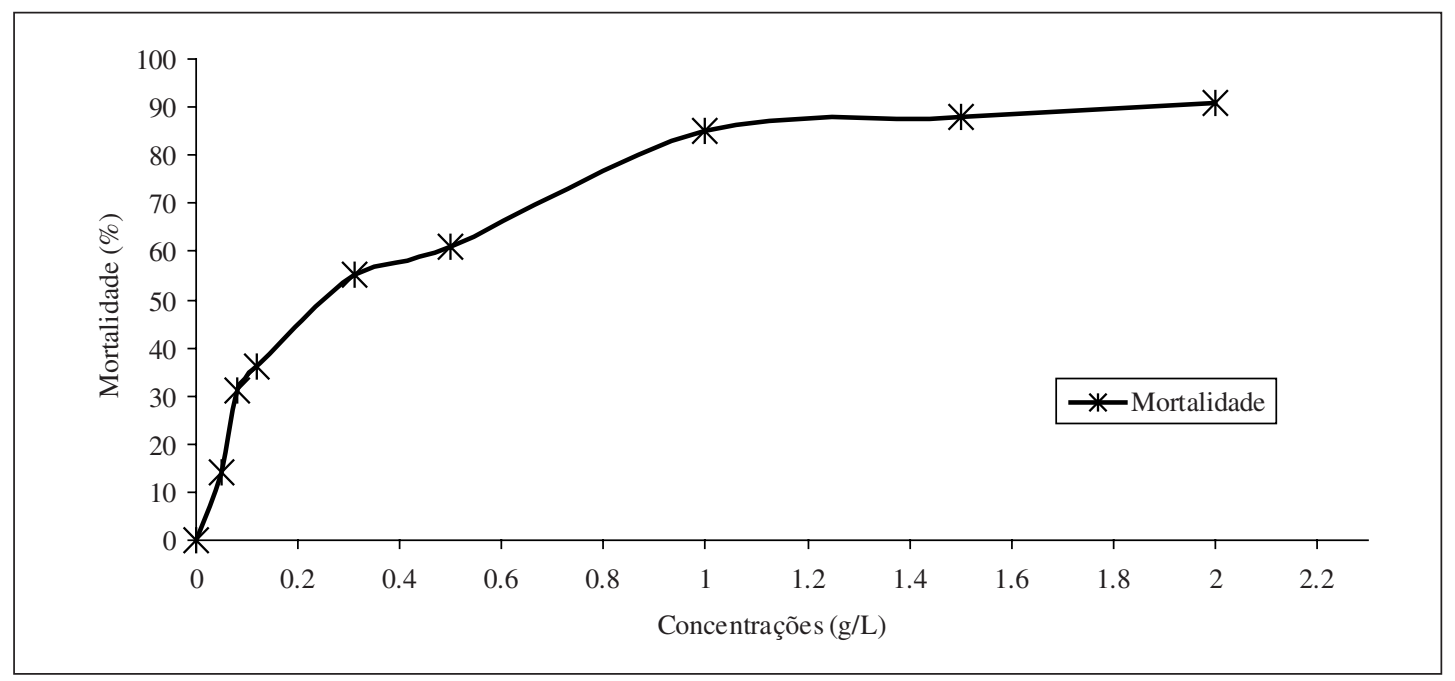

Gráfico 1. Mortalidade confirmada das ninfas de B. brassicae por B. bassiana em folhas de repolho. 


\section{CONCLUSÃO}

O isolado de B. bassiana contido no produto comercial, Boveril ${ }^{\circledR}$, é uma medida de controle eficiente para o manejo integrado do pulgão da

\section{REFERÊNCIAS BIBLIOGRAFICAS}

ALVES, E.B.; ALVES, S.B.; OMOTO, C.; CASARIN, N.F.B. 2004. Utilização de fungos entomopatogênicos em mistura com o acaricida dicofol no manejo da resistência de Brevipalpus phoenicis (Acari: Tenuipalpidae). In: XX Congresso Brasileiro de Entomologia, 2004, Gramado. XX Congresso Brasileiro de Entomologia. Londrina : Sociedade Entomológica do Brasil, v. 20. p. 515.

ALVES, S.B.; TAMAI, M.A.; ROSSI-ZALAF, L.S.; CASTIGLIONI, E. 2006. Patogenicidade de Beauveria bassiana ao ácaro da ferrugem dos citros (Phyllocoptruta oleivora). FUNDECITRUS (Fundo de Defesa da Citricultura), pesquisa do mês. Disponível em: http://www.fundecitrus. com.br/p_mes_br.html. Acesso em 30 jan. 2007.

ALVES, S.B.; MEDEIROS, M.B.; TAMAI, M.A.; LOPES, R.B. 2001. Trofobiose e microrganismos na proteção de plantas. Biotecnologia Ciência \& Desenvolvimento, v.21, p. 16-21.

ALVES, S.B.; ALMEIDA, J.E.M. 1997. Controle biológico das pragas das pastagens. In: Simpósio Sobre Ecossistema de Pastagens 3. Jaboticabal, SP. Anais. Jaboticabal: UNESP. v.3, p. 318-341.

ANDREI, E. 1996. Compêndio de defensivos agrícolas. $5^{\mathrm{a}}$ ed., São Paulo, Andrei, 506 p.

CATIE. 1990. Guia para el manejo integrado de plagas del cultivo de repolho. Turrialba. CATIE (Centro Agronómico Tropical de Investigación y Ensenãnza), p. 81.

ELLIS, P.R.; SINGH, R. 1993. A review of the host plants of the cabbage aphid, Brevicoryne brassicae (Homoptera, couve ( $B$. brassicae), sendo a concentração de $1,0 \mathrm{~g} / \mathrm{L}$ a mais eficiente para o controle dessa praga. Porém, as concentrações de 0,31 e $0,50 \mathrm{~g} / \mathrm{L}$ são as mais viáveis economicamente.

Aphididae). International Organisation for Biological and Integrated Control/ West Palaeartctic Regional Section (IOBC/WPRS Bulletin), v.16, p. 192-201.

GALLO, D.; NAKANO, O.; SILVEIRA NETO, S.; CARVALHO, R.P.L.; BAPTISTA, G.C.; BERTI FILHO, E.; PARRA, J.R.; ZUCCHI, R.A.; ALVES, S.B.; VENDRAMIN. J.D.; MARCHINI. J.D.; LOPES, J.R.S.; OMOTO, C. 2002. Pragas das Plantas e Seu Controle. In: Manual de Entomologia Agrícola. Piracicaba. FEALQ. cap. 12 , p. 722 .

LONGHINI, L.C.S.B.; BUSOLI, A.C. 1993. Controle integrado de Brevicoryne brassicae (L., 1758) (Homoptera: Aphididae) e Ascia monuste orseis (Latr., 1819) (Lepidoptera: Pieridae), em couve (Brassica oleraceae var. acephala). Científica, v.21, p. 231-237.

LOUREIRO, E.S.; MOINO, J.R.A. 2006. Patogenicidade de Fungos Hifomicetos aos Pulgões Aphis gossypii Glover e Myzus persicae (Sulzer) (Hemiptera: Aphididae). Neotropical Entomology, v.35, p. 660-665.

OMOTO, C.; DENNEHY, C.J.; MCCOY, C.W.; CRANE, S.E.; LONG, J.W. 1994. Detection and characterization of the interpopulation variation of citrus rust mite (Acari: Eriophyidae) resistance to dicofol in Florida citrus. Journal of Enconomic Entomology, v.87, p. 566-572.

ROBBS, C.F. BITTENCOURT, A.M. 1998. Controle Biológico de insetos nocivos a agricultura com o emprego de fungos imperfeitos ou hifomicetos. Biotecnologia Ciência \& Desenvolvimento, v.6, p. 10-12. 